\title{
Emotional Experience and the Mood-Congruent Working Memory Effect in First-Onset and Untreated Depressive Disorder Patients
}

\section{Li Mi1 ${ }^{1-3}$, Lu Shengfu ${ }^{1-3^{*}}$, Feng Lei ${ }^{4,5}$, Fu Bingbing ${ }^{4}$, Wang Gang ${ }^{4,6}$, Zhong Ning ${ }^{1-3,7^{*}}$ and Hu Bin ${ }^{8}$}

${ }^{1}$ Laboratory of Intelligent Science \& Technology, The International WIC Institute, Beijing University of Technology, Beijing, China

${ }^{2}$ Beijing International Collaboration Base on Brain Informatics and Wisdom Services, Beijing, China

${ }^{3}$ Beijing Key Laboratory of MRI and Brain Informatics, Beijing, China

${ }^{4}$ Mood Disorders Center \& China Clinical Research Center for Mental Disorders, Beijing Anding Hospital, Capital Medical University, Beijing, China

${ }^{5}$ Beijing Key Laboratory for Mental Disorders, Beijing, China

${ }^{6}$ Center of Depression, Beijing Institute for Brain Disorders, Beijing, China

${ }^{7}$ Department of Life Science and Informatics, Maebashi Institute of Technology Maebashi, Japan

${ }^{8}$ Ubiquitous Awareness and Intelligent Solutions Lab, Lanzhou University, Lanzhou, China

\begin{abstract}
Using an improved Sternberg working memory paradigm and affective pictures of different valences, this study investigates the emotional experience capability of 22 first-onset and untreated major depressive disorder patients (MDD) compared with the matching 22 healthy control participants $(\mathrm{HC})$ and whether there is a moodcongruent working memory effect. We employed a general linear model analysis of variance (ANOVA) with two-factor repeated measures analysis on the emotional experience capability (pupil diameter changes) and the working memory performance (accuracy). The results show that the pupil diameter changes of positive emotions are significantly greater in MDD than those in $\mathrm{HC}(\mathrm{p}<0.001)$, and the pupil diameter changes of negative emotions are not significantly different between two groups $(p=0.055)$, which suggest that MDD have a significantly decrease in the ability to experience pleasure (anhedonia). In addition, the results only present that the working memory performances of negative emotions are significantly greater in MDD than those of positive emotions $(p<0.05)$, which indicates that there is a mood-congruent memory effect. Moreover, in MDD, a positive correlation is found between the pupil diameter changes and working memory performances of positive emotions, however there is no correlation between those of negative emotions. Taken together, these results suggest that MDD have a moodcongruent memory effect and anhedonia, and the mood-congruent memory effect may be due to the decreased memory performances of positive emotions (a decrease in the ability to experience pleasure), but not those of negative emotions increased. This study not only illustrates that the core symptoms of depressive patients may be a mood-congruent memory effect and anhedonia but also enriches the connotation of anhedonia as an endophenotype indicator.
\end{abstract}

Keywords: First-onset and untreated depressive disorder; Working memory; Mood congruence; Emotional experience; Eye-tracking

\section{Introduction}

The working memory is a system that provides cognitive information processing with temporary information storage and limited processing; it is the cognitive basis for speech comprehension, learning, reasoning, and problem solving [1]. Previous research has shown a significantly diminished working memory in untreated patients with major depressive disorder [2], which manifests in significantly diminished working memory accuracy and significantly extended reaction time [3]. A study by Schatzberg et al. found that patients with major depressive disorder had significantly impaired attention and verbal memory [4]. Harvey et al. used an n-back working memory paradigm to study verbal memory and found that the working memory capacity of patients with major depressive disorder was significantly impaired compared to a healthy control (HC) group, manifesting in significantly reduced accuracy and a significantly slower memory retrieval capability [5]. The working memory results of Rose and Ebmeier [6], who used a symbolic n-back experimental paradigm on patients with major depressive disorder, were consistent with the results of Harvey et al. [5]. However, differently, the verbal memory-based study by Fossati et al. found that depressive patients had normal recognition and cued recall but impaired free recall [7].

Research on emotions and memory pays close attention to memory differences because of the different emotional valences between depressive patients and normal people; in addition, researchers also pay more attention to studies on the effect of mood on memory, particularly in depressive patients. In a working memory study by Bower et al. [8], subjects were asked to read a story with both sad and happy plots. Prior to reading, happy and sad emotions were induced in the subjects through hypnosis. After reading, the subjects were asked to recall the story in a neutral emotional state. The results found that the subjects who were in a sad mood while reading remembered more details from the sad story, but the subjects who were in a happy mood while reading remembered more details from the happy story. Subsequently, Blaney defined mood-congruent memory as "material of a similar emotional valence to the emotional state during encoding or retrieval facilitates encoding and retrieval" [9].

Mood-congruent working memory research on depressed patients

*Corresponding authors: Shengfu Lu, Laboratory of Intelligent Science \& Technology, The International WIC Institute, Beijing University of Technology, Beijing 100024, China, Tel: +86-10-6739-6667; E-mail: lusf@bjut.edu.cn

Ning Zhong, Department of Life Science and Informatics, Maebashi Institute of Technology Maebashi, Japan, Tel: +81-27-265-7366/027-265-7366; E-mail: zhong@maebashi-it.ac.jp

Received: May 16, 2016; Accepted: July 14, 2016; Published: July 20, 2016

Citation: Mi L, Shengfu L, Lei F, Bingbing F, Gang W, et al. (2016) Emotional Experience and the Mood-Congruent Working Memory Effect in First-Onset and Untreated Depressive Disorder Patients. J Psychiatry 19: 379. doi:10.4172/23785756.1000379

Copyright: (c) $2016 \mathrm{Mi} \mathrm{L}$, et al. This is an open-access article distributed under the terms of the Creative Commons Attribution License, which permits unrestricted use, distribution, and reproduction in any medium, provided the original author and source are credited. 
has shown that compared to an HC group, patients with major depression could remember more mood-congruent negative stimuli material but remembered less mood-incongruent positive stimuli material [10]. The working memory performance of unmedicated outpatients with major depression was significantly diminished compared to that of normal people [11], demonstrating not only that depressive patients are emotionally impaired but also that defects in working memory and other cognitive functions are concomitant $[5,12,13]$ and manifest as enhanced memory of mood-congruent negative material and diminished memory of mood-incongruent positive material; this is referred to as the mood-congruent memory effect. The moodcongruent working memory study on subjects in a natural emotional state (depressive patients) and subjects in an induced depressive emotional state by Kwiatkowski and Parkinson [14] showed that only depressive patients manifested a mood-congruent memory effect. The working memory study by Direnfeld and Roberts found [15] that the mood-congruent memory effect generated in patients with either major or minor depressive disorder (naturally depressed subjects) was better than that in subjects with induced depression. This showed that naturally depressed subjects had a negative cognitive schema, which was consistent with Beck's theory of depression on the cognitive schema [16]. Cognitive schema is a deep cognitive structure formed by an individual's past experiences that dominates the individual's information processing process (attention, selection, storage, and processing of information), which is to say that an individual tends to pay attention to, choose, and process information consistent with his own cognitive schema. According to the cognitive schema theory, depressive patients who are naturally depressed have a negative cognitive schema and prefer to pay attention to, choose, and perform refined processing on negative information [17], thus maintaining and developing their depressed mood. In this way, more focus is placed on mood-congruent information, and such information can better receive refined processing, thereby improving memory performance. However, some studies did not find a mood-congruent working memory effect. For example, Denny and Hunt did not find a mood-congruent working memory effect when depressed subjects performed a word fragment completion task, but they observed this phenomenon in an explicit free recall task [18]. Watkins used cued recall and word stem completion to conduct memory tests on depressed subjects, and they also did not find a mood-congruent working memory effect [19]. Erickson et al. used non-clinically depressed people and ordinary people as subjects to study mood-congruent working memory effect in a go/no-go task; the results found no significant differences in recall performance between the non-clinically depressed group and the HC group [20].

Anhedonia and a depressed mood are two important criteria in diagnosing major depressive disorder [21]. Anhedonia refers to a diminished capacity to experience pleasure; it is an endophenotype of major depressive disorder [22]. The study by Mathews and Barch showed that individuals with a high degree of anhedonia in a normal university student population had weakened assessment regarding the degree of pleasantness of positive words [23]. The study by Kaviani et al. indicated that depressive patients had decreased subjective experience with positive stimuli [24]. The prefrontal cortex plays a key role in the working memory and the processing pathways of emotions and rewards. Research has shown that abnormal activity in the prefrontal cortex leads to anhedonia [25], manifesting in a decreased level of activity in the left prefrontal lobe, which processes positive emotional information and is related to affective tendency, and an enhanced level of activity in the right prefrontal lobe, which processes negative emotions and is related to affective aversion [26].
Previous studies have indicated that pupil size changes could reflect the psychological expression related to emotional activities, which is embodied in mydriasis as a result of psychological activities generated by positive emotional stimuli and miosis as a result of psychological activities generated by negative emotional stimuli $[27,28]$. Hess conducted an experiment with drawings on faces that have no pupils to avoid the disturbance of light reflex. He found that participants drew larger pupils on the happy face than on the scowling face [29]. Hicks et al. through the study of 223 colleges using the same experimental method found the consistent results [30]. Emotional psychology believes that an emotional experience is an individual's subjective psychological feelings toward external emotional stimuli. Different emotional valences generate different psychological activities and feelings that are embodied as positive psychological feelings generated by positive emotional stimuli and negative psychological feelings generated by negative emotional stimuli. Therefore, in reality, the dilation and contraction in pupil size under external visual and emotional stimuli reflect the emotional experience from different external emotional stimuli.

Although Bower et al. firstly found that the emotional working memory has a mood-congruent memory effect; however it is still a debate on whether this effect exists in depression [8]. Up to now, many factors may be associated with these inconsistency results, for example, a state of mind, experiment material, and the inclusion criteria of depression. Taken together, although there are many studies that focus on the working memory of depressive patients, these studies have some inadequacies. First, there are inconsistencies regarding the presence of a mood-congruent working memory effect; second, there are more studies of working memory based on affective words but few studies of working memory based on affective pictures; third, in mood-congruent working memory research, more literature has focused on various emotional memories, but there is a lack of literature on the relationship between emotional experience and working memory performances; and the last, there is still no definite evidence about whether the depressed participants after antidepressant treatment such as taking medicine have an effect on working memory. This study focuses on first-onset and untreated depressive patients and uses positive, neutral, and negative affective pictures based on an improved Sternberg working memory paradigm. Although the emphasis is on the investigation of whether there is a mood-congruent working memory effect in first-onset and untreated depressive patients, this study also investigates the effect of emotional experience on working memory. Therefore, our hypotheses are: first, compared with HC, MDD would have a significantly decreased emotional experience on positive emotions, whereas a significantly increased emotional experience on negative emotions; Second, compared with HC, MDD's working memory capacity would be significantly reduced, showing significantly lower accuracy and longer reaction time; Third, MDD would have a mood-congruent working memory effect, that is the memory accuracy for negative affective pictures would be significantly higher than those for positive affective pictures.

\section{Methods}

\section{Participants}

Twenty-two first-onset and untreated depressive patients (MDD) and 22 matching (according to age, gender and education level of depressed patients) Health Control participants (HC) participated in this experiment. The MDD consisted of outpatients who had 
undergone first-time consultation at the Beijing Anding Hospital of Capital Medical University; the HC were recruited through posters. The MDD were screened by psychiatrists using the Mini-International Neuropsychiatric Interview (MINI) and were diagnosed using the Diagnostic and Statistical Manual of Mental Disorders, 4th Edition (DSM-IV). In addition to using a Quick Inventory of Depressive Symptomatology (Self-Report) (QIDS-SR16) for self-assessment, another physician administered a Hamilton.

Depression Rating Scale (HAMD) for evaluation. The inclusion criteria for the MDD group were as follows: (1) aged 18 to 60 years old, right-handed, in line with DSM-IV diagnostic criteria for firstonset depressive disorder; (2) without receiving any treatment or antidepressant drugs; (3) QIDS-SR16 (self-assessment based on the QIDS-SR16) >5, HAMD>7; and (4) no color blindness or other ocular disorder, normal vision or normal corrected vision, able to complete eye-tracking experiments. The exclusion criteria for the MDD were as follows: having other psychotic disorders according with the DSM-IV diagnostic, including schizoaffective disorder, schizophrenia, bipolar disorder, mental retardation, and pervasive developmental disorder; having severe symptoms of anxiety and panic disorders; having suicidal ideas or suicidal behavior; having mental disorders caused by the substance abuse (e.g., alcohol, drugs); having taken antidepressant drugs or other drugs for the treatment of mental illness on their own; having been treated by ECT; or having a history of epilepsy.

The selection criteria for the HC were as follows: (1) aged 18 to 60 years old, right-handed; (2) QIDS-SR16 $<5$, Beck depression inventory $(\mathrm{BDI})<4$; (3) having taken no medications that affect the functions of the neuropsychiatric system, no previous history of depressive disorder or other mental illness, no dependence on alcohol or other substances; and (4) no color blindness or other ocular disorder, normal vision or normal corrected vision. All of the participants signed the informed consent form and voluntarily participated in this experiment. After the completion of the experiment, the participants were compensated monetarily.

\section{Experimental methods}

This experiment used affective pictures as the stimuli for the task. Generally speaking, the use of affective pictures as stimuli for a task, compared to affective words, can transmit stronger emotional information and better induce emotions in the participants; thus, it is more favorable for testing the effects of various types of emotional information on the working memory. All of the affective pictures used in the experiment came from the International Affective Picture System (IAPS) [31]. There were 60 pictures in each of the three categoriesneutral, negative, and positive. Pictures with a high degree of pleasure $(7.31 \pm 0.44)$ and a high degree of arousal $(5.54 \pm 0.44)$ were used for the positive pictures. Pictures with a low degree of pleasure $(0.79 \pm 0.51)$ and a high degree of arousal $(5.97 \pm 0.44)$ were used for the negative pictures. Pictures with a moderate degree of pleasure $(5.18 \pm 0.17)$ and a low degree of arousal $(3.23 \pm 0.22)$ were used for the neutral pictures. To maintain consistency in the size, gray-scale, and resolution of all of the pictures, the software Picture Manager was used to provide unified treatment of the pictures.

\section{Experimental paradigm and procedures}

This experiment used an improved Sternberg working memory paradigm. To study the patients' emotional experience capability and whether there was a mood-congruent memory effect, each cue was prompted by using four pictures in the same category (four positive pictures, four negative pictures, or four neutral pictures); the four pictures corresponded to four different positions (upper left, upper right, lower left, and lower right). Thus, the cues used in the experiment included three categories: positive affective pictures, negative affective pictures, and neutral affective pictures. The probe stimulus picture was in the same category as the cued prompts and appeared in the center of the screen.

Experimental procedure: First, a "+" sign appeared in the middle of the screen for 0.5 seconds, prompting the subject on the immediate appearance of the cue. Then, the cue was shown for 10 seconds, letting the subject remember each picture in the cue. After 5 seconds of memory retention subsequent to the disappearance of the cue, a probe picture appeared in the center of the screen (the maximum present time is 3.5 seconds), letting the subject judge whether the probe picture had appeared in the preceding cue prompt. If it had appeared, then the subject pushed the left key; after the probe disappeared, a "*” sign appears after the key was pushed, prompting a 2-second rest before entering the next trial. Figure 1 shows a schematic representation of an example trial in our experiment.

During the experimental process, the Tobii T120 Eye Tracker was used to synchronically collect eye-tracking data on the participants while they were scanning the cue. The eye-tracking system recorded at a frequency of $120 \mathrm{~Hz}$ (coordinates were sampled every $8.333 \mathrm{~ms}$ ). The participants' eyes were kept at a distance of $60 \mathrm{~cm}$ from the screen. A five-point calibration was performed before each recording session. Eye movements that lasted for a minimum of $100 \mathrm{~ms}$ within a $1^{\circ}$ visual angle were classified as a fixation. Tobii Studio software (version 2.2.7) was used for the stimuli presentation and collection of visual fixation data.

\section{Statistical analysis}

Firstly, in the single-subject analysis, the working memory accuracy is the percentage of the number of correct tasks divided by the total number of tasks for one of the emotional categories (positive, negative

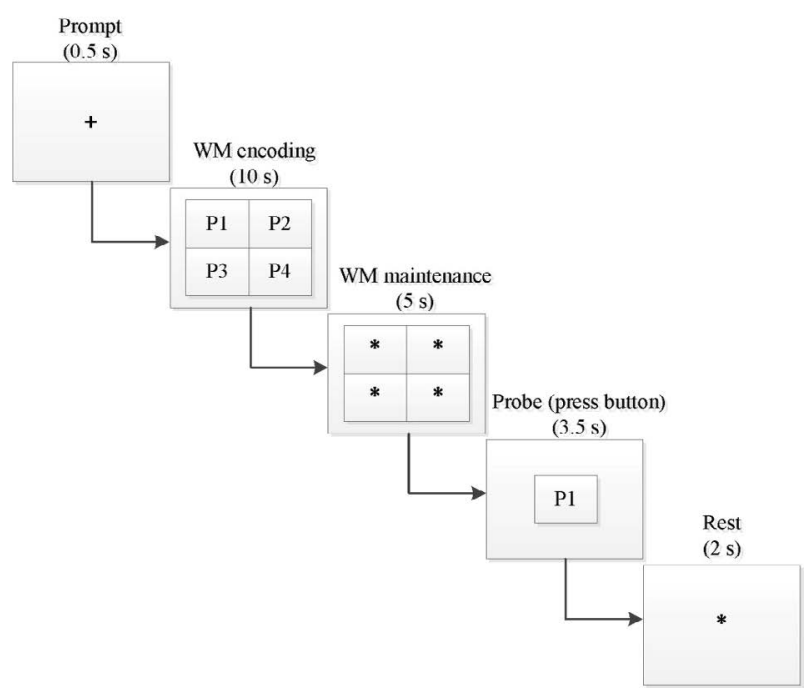

Figure 1: A schematic representation of an example trial of the working memory (WM) task in our experiment. Each trial included a symbol of "+" as a prompt ( 0.5 seconds); and a WM encoding task including the same emotional category of four pictures (10 seconds), in which p1, p2, p3 and p4 represent four positive affective pictures; and then followed by a WM maintenance task ( 5 seconds); and a position probe to ask the subjects to judge by pressing the buttons (the maximum present time is 3.5 seconds), followed by a 2-second rest. 
or neutral); and the response time is the total amount of time required to answer each task divided by the total number of tasks for one of the emotional categories.

Secondly, we employed a general linear model analysis of variance (ANOVA) with repeated measures analysis on the emotional experience capability (pupil size changes), working memory performances and mood congruent working memory effect. A 2 (groups: MDD, HC) $\times 2$ (emotional categories: positive, negative) mixed model was used for emotional experience capability. A 2 (groups: MDD, HC) $\times 3$ (emotional categories: positive, negative, neutral) mixed model was used separately to analyze whether there are significant differences between groups in the accuracy and reaction time. The mood-congruent analysis was mainly focused on whether there was a significant difference of intergroup between the memory accuracy of positive pictures and negative pictures. Thus, a 2 (groups, MDD, HC) $\times 2$ (emotional categories: positive, negative) mixed model was used for the moodcongruent analysis. In addition, in the above models, group was a between-subjects factor and emotional category was an internal factor of the participants.

Thirdly, a chi-square test was used to evaluate the intergroup difference in gender distribution, and an independent sample t-test was used to assess the intergroup differences in the age distribution and years of education distribution. After performing an analysis of variance on the mixed models in regard to intergroup or intragroup main effect significance or interaction significance, a pairwise t-test was performed (an independent sample t-test was used for intergroup effects, and a paired sample t-test was used for intragroup effects). SPSS 20.0 statistical analysis software was used to perform the statistical analysis.

At last, to test the reliability of the statistical check, in addition to estimating the size of the statistical effect (the main effect was partial eta squared, and the t-test used Cohen's d value), we also obtained Bayesian analysis results [32]. A Bayesian analysis could provide support for the probability of the null hypothesis, which provided evidence for us to further understand the reliability of statistical analysis in supporting the variance or mean equivalence hypothesis $(\mathrm{H} 0)$.

\section{Results}

\section{Demographic and clinical data}

As shown in Table 1, there was no significant difference in the gender distribution between the two groups $\left(\chi^{2}=0.091, \mathrm{p}=0.763\right)$; there was no significant difference in the age distribution between the two groups [ $t(42)=0.136, p=0.893, d=0.043$ ]; there was no significant difference in the education level distribution between the two groups [ $\mathrm{t}(42)=-1.146, \mathrm{p}=0.258, \mathrm{~d}=-0.355]$; there was a significant difference in the QIDS-SR16 between the two groups [ $t(42)=20.002, p=0.000$, $\mathrm{d}=6.174]$; and the average HAMD score of the MDD was 21.05 \pm 3.97 .

\section{Comparison of emotional experience between the two groups}

The analysis of variance on pupil size changes found that the intergroup main effect of pupil size changes was significant $[F$ $\left.(1,42)=21.650, \quad \mathrm{p}<0.001, \quad \eta^{2}=0.340\right]$. Emotional stimuli induced significantly greater pupil size changes in the HC group than in the MDD group, and the interaction of the emotional category * group was significant $\left[F(1,42)=12.566, p<0.05, \eta^{2}=0.230\right]$. The main effect of the emotional category was significant $[F(1,42)=59.556, p<0.001$, $\left.\eta^{2}=0.340\right]$. The pairwise intergroup comparison results are shown in Figure 2. Pupil size changes from positive emotions in the MDD

\begin{tabular}{|c|c|c|c|}
\hline & MDD Group (n=22) & HC Group (n=22) & p-value \\
\hline $\begin{array}{c}\text { Gender } \\
\text { (Males:Females) }\end{array}$ & $10: 12$ & $11: 11$ & 0.763 \\
\hline Age (years) & $34.14 \pm 9.36$ & $33.77 \pm 8.37$ & 0.893 \\
\hline Education level (years) & $13.36 \pm 3.44$ & $14.50 \pm 3.13$ & 0.258 \\
\hline QIDS-SR16 & $16.55 \pm 3.10$ & $2.41 \pm 1.18$ & 0 \\
\hline HAMD (17-item) & $21.05 \pm 3.97$ & & \\
\hline
\end{tabular}

Table 1: Comparison of intergroup demographic differences $(M \pm S D)$.

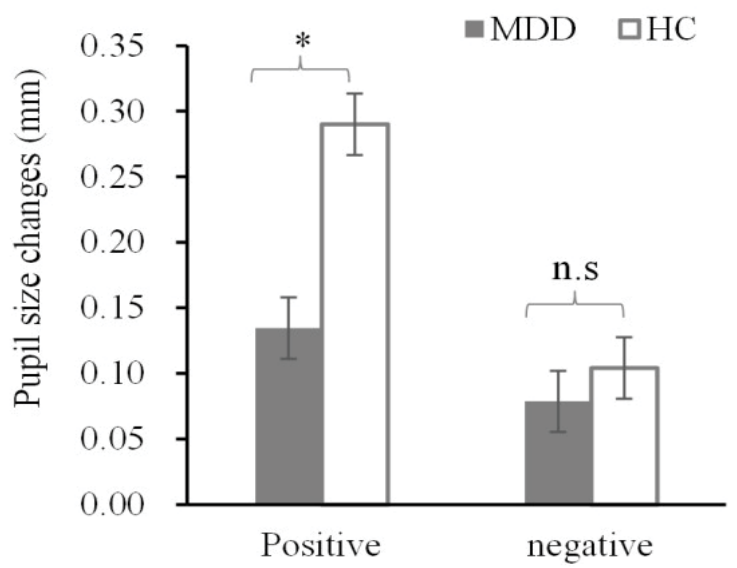

Figure 2: Comparison of pupil size changes induced by affective pictures of different valences between the two groups. *: $p<0.05$; n.s., no significant difference.

group were significantly less compared to those in the HC group [ $\mathrm{t}(42)=-5.845, \mathrm{p}<0.05, \mathrm{~d}=-1.804$; PBayes $(\mathrm{H} 0)=0.999$ ], indicating that, compared to the HC, the MDD clearly had insufficient positive emotional experiences. There was no significant difference in pupil size changes from negative emotions between the MDD group and the HC group [ $\mathrm{t}(42)=-1.974, \mathrm{p}=0.055, \mathrm{~d}=-0.310$; PBayes $(\mathrm{H} 0)=0.580$ ], indicating that there was no significant difference between MDD and $\mathrm{HC}$ regarding negative emotional experiences.

\section{Analysis of mood-congruent working memory effect}

A mixed-model ANOVA with 2 (groups: MDD, HC) $\times 3$ (emotional categories: positive, neutral, negative) analysis on the working memory accuracy showed that the group factor has no significant effect $[\mathrm{F}$ $\left.(1,42)=3.829, p=0.057, \eta^{2}=0.084\right]$, the emotional categorical factor had a significant effect $\left[F(1,42)=10.031, p<0.05, \eta^{2}=0.193\right]$, and the interaction of the group * emotional category was significant $[\mathrm{F}$ $\left.(1,42)=5.671, \mathrm{p}<0.05, \eta^{2}=0.119\right]$. To further analyze the differences in emotional categories, a pairwise t-test analysis (Figure 3 ) showed that, in the MDD group, the working memory accuracy of negative affective pictures was significantly higher than that of positive affective pictures $[\mathrm{t}(21)=2.821, \mathrm{p}<0.05, \mathrm{~d}=0.822$, PBayes $(\mathrm{H} 0)=0.170]$; in the HC group, there was no significant differences between them $[\mathrm{t}(21)=0.810$, $\mathrm{p}=0.427, \mathrm{~d}=0.259$, PBayes $(\mathrm{H} 0)=0.770]$. The results suggest that the mood-congruent memory effect exists in the MDD group, showing that the working memory accuracy of negative affective pictures consistent with the MDD's mood is significantly higher than that of positive affective pictures.

\section{Intragroup comparison of working memory performances}




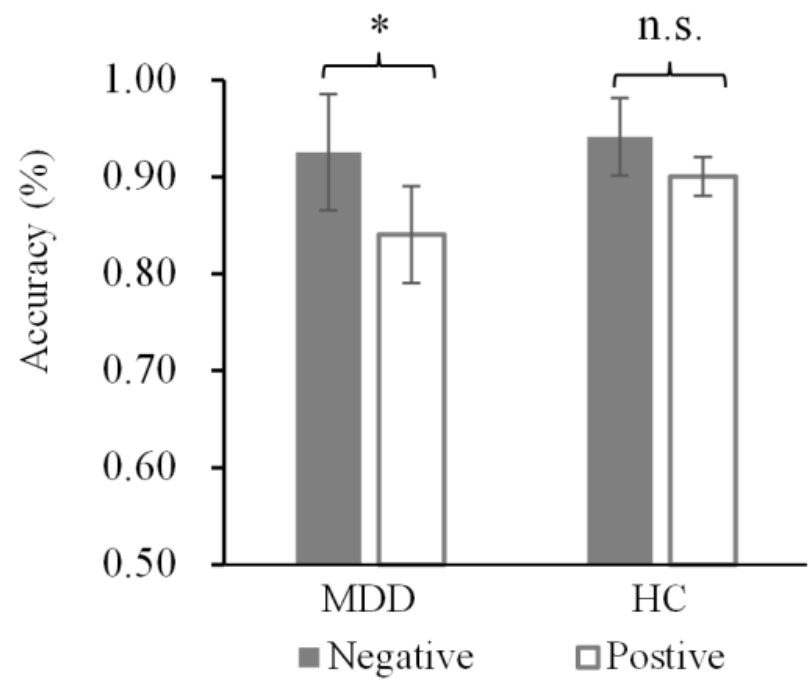

Figure 3: Analysis of mood-congruent working memory effect. *: $p<0.05$; n.s., no significant difference.

\begin{tabular}{|c|c|c|c|}
\hline \multirow{2}{*}{ Group } & $\begin{array}{c}\text { Emotional } \\
\text { Category }\end{array}$ & Accuracy (\%) & Response Time (ms) \\
\hline \multirow{3}{*}{ MDD } & Positive & $0.84 \pm 0.13$ & $1995.05 \pm 786.19$ \\
\cline { 2 - 4 } & Negative & $0.93 \pm 0.06$ & $1670.36 \pm 484.96$ \\
\cline { 2 - 4 } & Neutral & $0.91 \pm 0.07$ & $1548.68 \pm 576.59$ \\
\hline \multirow{2}{*}{ HC } & Positive & $0.90 \pm 0.09$ & $1127.45 \pm 273.44$ \\
\cline { 2 - 4 } & Negative & $0.94 \pm 0.04$ & $1498.95 \pm 354.88$ \\
\cline { 2 - 4 } & Neutral & $0.93 \pm 0.05$ & $1320.09 \pm 271.18$ \\
\hline
\end{tabular}

Table 2: Statistical results of working memory accuracy and response time.

The working memory accuracy and response time are shown in Table 2. For the working memory accuracy, a mixed-model ANOVA with 2 (groups: MDD, HC) $\times 3$ (emotional categories: positive, neutral, negative) analysis showed that the group factor has no significant effect $\left[F(1,42)=2.901, p=0.096, \eta^{2}=0.065\right]$, the emotional categorical factor had a significant effect $\left[\mathrm{F}(2,84)=2.052, \mathrm{p}<0.05, \eta^{2}=0.107\right]$, and the interaction of the group ${ }^{*}$ emotional category was not significant $\left[F(2,84)=1.746, p=0.181, \eta^{2}=0.040\right]$, which suggest that the MDD's memory encoding and memory retention are not impaired.

For the working memory response time, a mixed-model ANOVA with 2 (groups: MDD, HC) $\times 3$ (emotional categories: positive, neutral, negative) analysis showed that the group factor has a significant effect $\left[F(1,42)=10.920, p<0.05, \eta^{2}=0.206\right]$, the emotional categorical factor had a significant effect $\left[F(2,84)=24.563, p<0.001, \eta^{2}=0.369\right]$, and the interaction of the group ${ }^{*}$ emotional category was significant [F $\left.(2,84)=4.496, p<0.05, \eta^{2}=0.073\right]$. The intragroup pairwise $t$-test analysis showed that, for the positive and negative affective pictures, the MDD was significantly longer than the HC [positive: $t(42)=2.698$, $\mathrm{p}<0.05, \mathrm{~d}=0.833$, PBayes $(\mathrm{H} 0)=0.831$; negative: $\mathrm{t}(42)=33.127, \mathrm{p}<0.001$, $\mathrm{d}=1.412$, PBayes $(\mathrm{H} 0)=1.000$ ]; for the neutral affective pictures, there was no significant difference between MDD and HC $[\mathrm{t}(42)=1.683$, $\mathrm{p}=0.100, \mathrm{~d}=0.519$, PBayes $(\mathrm{H} 0)=0.522]$. The results suggest that the affective pictures (whether it is positive or negative) has a significantly effect on the memory retrieval.

\section{Analysis of the relationship between emotional experience and working memory accuracy}

A series of Pearson correlation analyses were performed to investigate whether there was a correlation between emotional experience and the corresponding accuracy. Table 3 shows the correlation coefficients. The analysis indicated that there was only one significant relationship: the emotional experience of depressive patients under positive emotional stimuli showed a positive correlation with the positive emotional memory accuracy. No other significant correlation was identified.

\section{Discussion}

We studied the emotional experience of depressive patients from affective pictures of different valences, and the mood-congruent working memory effect through an eye-tracking experiment. The emotional experience results showed that the depressive disorder group had a significantly lower emotional experience capability for positive emotions compared to the HC group, indicating that depressive patients had a significantly diminished ability to experience pleasure and present obvious symptoms of anhedonia. However, there was no significant difference compared to the $\mathrm{HC}$ group in regard to emotional experience of negative emotions. The results partly support our first hypothesis that compared with HC, MDD would have a significantly decreased emotional experience on positive emotions, however a significantly increased emotional experience on negative emotions was not found. Our study results are supported by the results of Heller's study [33]; that is, emotional impairment in depressive patients manifests through diminished capabilities in processing positive emotions and maintaining brain activity but maintains the normal processing of negative emotions and maintenance of brain activity. Some studies also showed that regardless of whether the patient had major depressive disorder, their subjective experience of positive emotional stimuli was reduced [24]. The commonality between our study and the previous studies was a tendency to show that depressive patients (regardless of the severity of the depressive disorder symptoms) had anhedonia; inconsistent points were related to negative emotion processing and negative emotional experience. Our study results indicated that although there was no significant difference in negative emotional experience between the depressive patients and the HC group, the difference was marginally significant, and the Bayesian analysis results supported this conclusion with very weak evidence [34]. This experimental result may have been generated because of our lack of differentiation between the levels of severity of depressive disorder, or perhaps pupil size changes induced by different emotions more realistically reflected the individuals' psychological feelings and emotional experience. Therefore, future research should further determine whether there is a difference among depressive patients with different levels of depression severity in their emotional experience of negative emotional stimuli.

The second hypothesis is, compared with HC, MDD's working memory performance would be significantly reduced, showing significantly lower accuracy and longer reaction time. In this study,

\begin{tabular}{|c|c|c|}
\hline Group & Pupil Size Changes & Accuracy (\%) \\
\hline \multirow{2}{*}{ MDD } & Positive & $0.409^{*}$ \\
\cline { 2 - 3 } & Negative & -0.319 \\
\hline \multirow{2}{*}{ HC } & Positive & 0.185 \\
\cline { 2 - 3 } & Negative & -0.155 \\
\hline
\end{tabular}

Table 3: Correlation between emotional experience and corresponding accuracy $(\mathrm{p}<0.05)$. 
compared to the $\mathrm{HC}$ group, there was no abnormality in the working memory accuracy of the depressive disorder group. This result at least showed normal emotional working memory encoding and retention in depressive patients. Although the results of memory accuracy do not accord with the hypothesis, however there are still some previous studies supported our results, for example, previous research has found that individuals with depressive disorder were relatively undamaged in terms of various cognition measures [35]; The word-based working memory research by Fossati et al. also did not find cued recall impairment in those hindered by depression [7]. Some studies have found that the working memory performance of depressive patients was impaired $[2,5,6]$. The reason for these inconsistent results is that, in this study, to investigate the working memory performance of depressive patients, firstly, we used pictures instead of words because vocabulary is more closely linked with educational level than pictures; this way, the influence of educational-level factors could be excluded as much as possible; secondly, some studies thought that the working memory performance of depressive patients would diminish along with an increase in task difficulty [5,36,37]. Memory difficulty is one of the main factors that influence memory performance. The size of an individual's memory capacity has a relatively large effect on working memory performance. An individual's short-term memory capacity ranges from five to nine (seven \pm two) chunks. We used four affective pictures as memory cues, which is lower than the lower limit of memory capacity (five chunks), to reduce the influence of memory difficulty factors on memory performance as much as possible. Therefore, our study found that, on the basis of excluding the effects of educational level and memory difficulty as much as possible, the working memory encoding of affective pictures in depressive patients was not impaired. However, compared with HC, MDD's working memory response time of affective pictures (positive and negative) was significantly longer, whereas there was no significant difference between the two groups for the neutral affective pictures. The results indicate that both positive and negative affective information have seriously effect on the memory retrieval process, causing the memory retrieval slower, which is consistent with the results of Harvey [5], Rose and Ebmeier [6] using an n-back working memory paradigm on word memory. These studies all find that MDD's working memory retrieval time is significantly longer than the HC. Previous studies have shown that the posterior parietal cortex is associated with attention, episodic memory and working memory $[38,39]$. The posterior parietal cortex includes the dorsal parietal cortex (DPC) and ventral parietal cortex (VPC) [40]. Evidence suggests that DPC allocates effortful top-down attention for memory retrieval during cued retrieval, whereas VPC mediates spontaneous bottom-up memory retrieval during un-cued retrieval; in addition, activity in the DPC network has been associated with faster cued retrieval response times [41]. In the cued working memory test conducted in the present study, impaired DPC functions and functional connectivity disorders between DPC and MTL (medial temporal lobe) likely resulted in an inadequate top-down allocation of attention resources [41], thereby contributing to significantly prolonged memory retrieval time among the patients. However, depressed patients typically present with dysthymia symptoms due to their automatic retrieval and processing of unconscious negative events, which involves a bottomup, un-cued memory retrieval process of the VPC. Although cued and non-cued memory retrieval involves different posterior parietal cortexes, however, as additional resources are needed for processing the emotional information, cognitive controls cannot reasonably allocate time resources, leaving inadequate attention resources for cued memory retrieval. Consequently, patients' memory retrieval time are significantly delayed.

Our study supports the third hypothesis that the existence of a mood-congruent memory effect in depressive patients. That is, the memory accuracy of negative affective pictures was significantly greater than that of positive affective pictures in the MDD group, but there was no such significant difference found in the HC group. There may be three possible explanations for the mood-congruent memory effect in depressive patients: First, because the maintaining of depressive state is related to increasing negative cognition and contemplation $[42,43]$, the internal concentration of unconscious negative processing would use cognitive resources (particularly the resource of attention). The occupation of these limited resources would affect the processing of positive information, leading to a drop in memory performance on positive emotional information. Another possibility is that depressive patients may have a different cognitive schema from psychologically healthy people. Depressive patients have formed a negative cognitive schema in a chronic stress [44] or learned helplessness [45] environment, leading depressive patients to have a psychological conflict between processing positive external information stimuli and negative cognition, thereby affecting memory performance on positive information. Third, depressive patients have impaired attention, manifesting as insufficient attention to positive information and an attention bias for negative information $[17,46]$. Some studies have thought that non-depressed individuals have an attention preference for positive information as a "protective bias," but depressive patients lack this "protective bias" $[47,48]$. Therefore, decreased attention to positive stimuli not only causes a lack of positive emotions [49] but also leads to lower memory performance on positive emotional information.

We also measured pupil size changes induced by different emotional stimuli and the relationship with corresponding emotional working memory performance. We only found a positive correlation between positive pupil size changes in depressive patients and positive accuracy; we did not find any other significant correlations. This result may indicate a close relationship between the anhedonia severity level and the memory accuracy on positive information, manifesting as increasingly low memory accuracy on positive information as anhedonia becomes more severe. To a certain degree, this enriched the connotation of anhedonia as an endophenotype indicator. Previous studies have shown that for the positive emotional information, depressed patients' brain processing ability and brain activity are decreased, whereas for the negative emotional information, those can be maintained normally $[24,33]$. This may be the reason that the depressed patients' emotional experience ability and the working memory accuracy of positive emotional information are reduced; however, those of negative emotional information didn't show an abnormality. This also may give an explanation that the emotional experience of depressed patients under positive emotional stimuli showed a positive correlation with the positive emotional memory accuracy; however the negative emotional experience is not relevant to the negative emotional memory accuracy. In addition, HC's emotional experience and emotional memory abilities both have not shown abnormality, which may be the reason that HC's positive emotional experience ability is not relevant to the memory accuracy of positive emotional information. Otherwise, according to our experimental results, it may also suggest that the reason of depressed patients' mood-congruent memory effect is not the increased memory accuracy of negative emotional information, but it is because of the decreased memory accuracy of positive emotional information.

\section{Conclusion}


In conclusion, based on a group of first-onset and untreated depressive patients and an HC group that had never suffered from any mental illnesses as participants, we investigated the MDD's working memory of different affective pictures and their emotional experience. Our results suggest that (1) there is a mood-congruent memory effect in MDD, that is, the MDD can easily remember the negative stimuli which are consistent with their negative moods, whereas it is difficult to remember the positive stimuli; (2) anhedonia in depressed patients, i.e., a significant reduction in the ability to experience pleasure. And found that the positive emotional experience ability has a positive correlation with the working memory accuracy of positive emotions. Our result not only shows that the mood-congruent working memory effect and anhedonia are the core symptoms of depression; meanwhile, it enriches the connotation of anhedonia as an indicator of endophenotype. We selected first-onset and untreated depressive patients to test whether a mood-congruent memory effect is present in depressive disorder. It mainly considered prior research in which patients who were included had undergone various levels of medication and treatment, which, to a certain degree, would influence the patients' emotional experience and memory. First-onset and untreated depressive patients have not been "polluted" by medication and can more realistically reflect the emotional state and cognitive ability of depressive patients. This resulted in a limited number of first-onset and untreated depressive patients from the outpatient pool, leading to a relatively insufficient number of included patients within a given period. Its limitation is that there was only limited power in detecting differences between the patient group and the HC group. Although similar sample sizes have been used in other studies [17,50], studies with larger samples characterized by a greater severity of symptoms could reveal stronger group differences in emotional experience and emotional working memory with regards to positive and negative emotional information. A further limitation of this study is that our results are based on cross-sectional data; therefore, we are unable to draw conclusions about causality. In addition, no investigation on the differences between treated and untreated depressed patients in working memory performance is another limitation, which may affect the further explanation to the results. Finally, this study only focused on the working memory of mood-congruent effect, but not refers to the long-term memory.

\section{Acknowledgment}

This work is supported by the National Basic Research Pro-gram of China (2014CB744600), the Beijing Natural Science Foundation (4164080), the Beijing Outstanding Talent Training Foundation (2014000020124G039), the Internationa Science \& Technology Cooperation Program of China (2013DFA32180), the National Natural Science Foundation of China (61420106005), the Grantin-Aid for Scientific Research (C) from Japan Society for the Promotion of Science (26350994), the Beijing Municipal Science and Technology Project (D12100005012003), the Beijing Mu-nicipal Administration of Hospitals Clinical Medicine De-velopment of Special Funding (ZY201403), and the Beijing Municipal Science and technology achievement transfor-mation and industrialization projects funds (Z121100006112057).

\section{Competing interests}

The authors declare that they have no competing interests.

\section{References}

\section{Baddeley $A$ (1992) Working memory. Science 255: 556-559.}

2. Pelosi L, Slade T, Blumhardt LD, Sharma VK (2000) Working memory dysfunction in major depression: An event-related potential study. Clin Neurophysiol 111: 1531-1543.

3. Ortiz T, Pérez-Serrano JM, Zaglul C, Coullaut R, Coullaut J Jr, et al. (2003) Deficit of cognitive event-related potentials during a working task in patients with major depression. Actas Esp Psiquiatri 31: 177-181.
4. Schatzberg AF, Posener JA, DeBattista C, Kalehzan BM, Rothschild AJ, et al (2000) Neuropsychological deficits in psychotic versus nonpsychotic major depression and no mental illness. Am J Psychiat 157: 1095-1100.

5. Harvey PO, Le Bastard G, Pochon JB, Levy R, Alliaire JF, et al. (2004) Executive functions and updating of the contents of working memory in unipolar depression. J Psychiatr Res 38: 567-576.

6. Rose E J, Ebmeier K P (2006) Pattern of impaired working memory during major depression. J Affect Disorders 90: 149-161.

7. Fossati P, Coyette F, Ergis AM, Alliaire JF (2002) Influence of age and executive functioning on verbal memory of inpatients with depression. J Affect Disorders 68: 261-271.

8. Bower GH (1981) Mood and memory. Am Psychol 36: 129-148.

9. Blaney PH (1986) Affect and memory: A review. Psychol Bull 99: 229-246.

10. Matt G E, Vazquez C, Campbell W K (1992) Mood-congruent recall of affectively toned stimuli - a meta-analytic review. Clin Psychol Rev 12: 227-255.

11. Den Hartog HM, Derix MM, Van Bemmel AL, Kremer B, Jolles J (2003) Cognitive functioning in young and middle-aged unmedicated out-patients with major depression: Testing the effort and cognitive speed hypotheses. Psychol Med 33: 1443-1451.

12. Weiland-Fiedler P, Erickson K, Waldeck T, Luckenbaugh DA, Pike D, et al (2004) Evidence for continuing neuropsychological impairments in depression. J Affect Disorders 82: 253-258.

13. Harvey PO, Fossati P, Pochon JB, Levy R, Lebastard G, et al. (2005) Cognitive control and brain resources in major depression: An fMRI study using the n-back task. Neuroimage 26: 860-869.

14. Kwiatkowski SJ, Stanley RP (1994) Depression, elaboration, and mood congruence: Differences between natural and induced mood. Mem Cognit 22 225-233.

15. Direnfeld DM, Roberts JE (2006) Mood congruent memory in Dysphoria: The roles of state affect and cognitive style. Behav Res Ther 44: 1275-1285.

16. Beck AT (2002) Cognitive models of depression. In: Leahy R, Dowd ET (eds) Clinical Advances in Cognitive Psychotherapy: Theory and Application, Springer Publishing Company Inc, New York, USA.

17. Duque A, Vazquez C (2015) Double attention bias for positive and negative emotional faces in clinical depression: Evidence from an eye-tracking study. $J$ Behav Ther Exp Psychiat 46: 107-114.

18. Denny EB, Hunt RR (1992) Affective valence and memory in depression Dissociation of recall and fragment completion. J Abn Psychol 101: 575-580

19. Watkins PC, Vache K, Verney SP, Muller S, Mathews A (1996) Unconscious mood-congruent memory bias in depression. J Abn Psychol 105: 34-41.

20. Erickson K, Drevets WC, Clark L, Cannon DM, Bain EE, et al. (2005) Mood congruent bias in affective Go/No-Go performance of unmedicated patients with major depressive disorder. Am J Psychiat 162: 2171-2173.

21. American Psychiatric Association (1994) Diagnostic and statistical manual of mental disorders. 4th (edn) DSM-IV.

22. Hasler G, Drevets WC, Manji HK, Charney DS (2004) Discovering endophenotypes for major depression. Neuropsychopharmacol 29: 1765-1781.

23. Mathews JR, Barch DM (2006) Episodic memory for emotional and non emotional words in individuals with anhedonia. Psychiat Res 143: 121-133.

24. Kaviani H, Gray JA, Checkley SA, Raven PW, Wilson GD, et al. (2004) Affective modulation of the startle response in depression: Influence of the severity of depression, anhedonia, and anxiety. J Affect Disorders 83: 21-31.

25. Pizzagalli DA, Oakes TR, Fox AS, Chung MK, Larson CL, et al. (2009) Functional but not structural subgenual prefrontal cortex abnormalities in melancholia. Mol Psychiatry 9: 393-405.

26. Pizzagalli DA, Bogdan R, Ratner KG, Jahn AL (2007) Increased perceived stress is associated with blunted hedonic capacity: Potential implications for depression research. Behav Res Ther 45: 2742-2753.

27. Hess EH, Polt JM (1960) Pupil size as related to interest value of visual stimuli. Science 132: 349-350.

28. Hess EH (1965) Attitude and pupil size. Sci Am 212: 46-54. 
Citation: Mi L, Shengfu L, Lei F, Bingbing F, Gang W, et al. (2016) Emotional Experience and the Mood-Congruent Working Memory Effect in FirstOnset and Untreated Depressive Disorder Patients. J Psychiatry 19: 379. doi:10.4172/2378-5756.1000379

29. Hess EH (1975) The tell-tale eye. Van Nostrand Reinhold, New York, USA.

30. Hicks RA, Williams S L, Ferrante F (1979) Pupillary attributions of college students to happy and angry faces. Percept Mot Skills 48: 401-402.

31. Lang PJ, Bradley MM, Cuthbert BN (1997) International affective picture System (IAPS): Technical Manual and Affective Ratings. NIMH Center for the Study of Emotion and Attention.

32. Rouder J, Speckman PL, Sun D, Morey RD, Iverson G (2009) Bayesian t tests for accepting and rejecting the null hypothesis. Psychon Bull Rev 16: 225-237.

33. Heller AS, Johnstone T, Shackman AJ, Light SN, Peterson MJ, et al. (2009) Reduced capacity to sustain positive emotion in major depression reflects diminished maintenance of fronto-striatal brain activation. PNAS 106: 2244522450.

34. RafteryAE (1995) Bayesian model selection in social research. Sociol Methodo 25: 111-163.

35. Stein M, Miller AH, Trestman RL (1991) Depression, the immune system, and health and illness: Findings in search of meaning. Arch Gen Psychiat 48: 171-177.

36. Elderkin-Thompson V, Kumar A, Bilker WB, Dunkin JJ, Mintz J, et al. (2003) Neuropsychological deficits among patients with late-onset minor and major depression. Arch Clin Neuropsychol 18: 529-549.

37. Monks PJ, Thompson JM, Bullmore ET, Suckling J, Brammer MJ, et al. (2004) A functional MRI study of working memory task in euthymic bipolar disorder: Evidence for task-specific dysfunction. Bipolar Disord 6: 550-564.

38. Posner MI, Petersen SE (1990) The attention system of the human brain. Ann Rev Neurosci 13: 25-42.

39. Berryhill ME (2012) Insights from neuropsychology: pinpointing the role of the posterior parietal cortex in episodic and working memory. Front Integr Neurosci 6: 31

40. Cabeza R, Ciaramelli E, Olson IR, Moscovitch M (2008) The parietal cortex and episodic memory: an attentional account. Nat Rev Neurosci 9: 613-625.
41. Burianová H, Ciaramelli E, Grady CL, Moscovitch M (2012) Top-down and bottom-up attention-to-memory: mapping functional connectivity in two distinct networks that underlie cued and uncued recognition memory. Neuroimage 63: 1343-1352.

42. Riso LP, du Toit PL, Blandino JA, Penna S, Dacey S, et al. (2003) Cognitive aspects of chronic depression. J Abnorm Psychol 121: 72-80.

43. Siegle GJ, Steinhauer SR, Thase ME (2004) Pupillary assessment and computational modeling of the Stroop task in depression. Int J Psychophysio 52: 63-76.

44. Grippo AJ, Francis J, Beltz TG, Felder RB, Johnson AK (2005) Neuroendocrine and cytokine profile of chronic mild stress-induced anhedonia. Physiol Behav 84: 697-706.

45. Henn FA, Vollmayr B (2005) Stress models of depression: Forming genetically vulnerable strains. Neurosci Biobehav Rev 29: 799-804.

46. Teachman BA, Joormann J, Steinman SA, Gotlib IH (2012) Automaticity in anxiety disorders and major depressive disorder. Clin Psychol Rev 32: 575603.

47. Mccabe SB, Gotlib IH (1995) Selective attention and clinical depression: Performance on a deployment-of-attention task. J Abnorm Psychol 104: 241 245

48. Shane MS, Peterson JB (2007) An evaluation of early and late stage environmental processing of positive and negative information in dysphoria Cognition Emotion 21: 789-815.

49. Watson D, Naragon-Gainey K (2010) On the specificity of positive emotional dysfunction in psychopathology: Evidence from the mood and anxiety disorders and schizophrenia/schizotypy. Clin Psychol Rev 30: 839-848.

50. Mogg K, Millar N, Bradley BP(2000) Biases in eye movements to threatening facial expressions in generalized anxiety disorder and depressive disorder. $J$ Abnorm Psychol 109: 695-704. 